\title{
Artificial quantum photosynthetic materials
}

Received 00th January 20xx, Accepted 00th January 20xx DOI: $10.1039 / \times 0 \times x 00000 x$
Sabrina L. Slimani ${ }^{a}$, Roman Kostecki ${ }^{a}$, Ahmed Nuri Kursunlu ${ }^{b}$, Tak W. Kee ${ }^{c}$, Patrick C. Tapping ${ }^{c}$, Adrian M. Mak ${ }^{d}$ and James Q. Quach ${ }^{\text {a }}$

Photosynthesis has been shown to be a highly efficient process for energy transfer in plants and bacteria. It has been proposed that quantum mechanics plays a key role in this energy transfer process. There has been evidence that photosynthetic systems may exhibit quantum coherence. As artificial light-harvesting complexes have been proposed to mimic photosynthesis, it is prudent that artificial photosynthetic materials should also be tested for quantum coherence. To date, such studies have not been reported. In this work, we examine one such system, the BODIPY light harvesting complex (LHC), which has been shown to exhibit classical energy transfer via Förster resonance energy transfer. We compare the photon absorption of the LHC with the BODIPY chromophore by performing UV-visible, transient absorption, broadband pump-probe (BBPP) and two-dimensional electronic (2DES) spectroscopy. The 2DES and BBPP show evidence for quantum coherence, with oscillation frequencies of $100 \mathrm{~cm}^{-1}$ and $600 \mathrm{~cm}^{-1}$, which are attributable to vibronic, or exciton-phonon type coupling. Further computational analysis suggests strong couplings of the molecular orbitals of the LHC resulting from the stacking of neighbouring BODIPY chromophore units may contribute to undesirable hypochromic effects.

\section{Introduction}

Photosynthesis is the transformation of light energy to chemical energy and is mediated by light-harvesting complexes (LHCS). The LHCs present in plants and some bacteria are composed of chromophores, which upon photon absorption are excited from a ground state to an electronic excited state. ${ }^{1}$ 2 The energy from the excitation travels amongst the chromophores to the reaction centre where charge separation is initiated with efficiencies between $84 \%$ and $90 \% .3,4$ This efficiency represents the fraction of the donor chromophores de-excited via energy transfer to the acceptor chromophores. ${ }^{5}$ The energy transfer occurs via FRET.2, 5 Energy transfer is affected by the distance between the chromophores, the spectral overlap between the fluorescence emission of the donor chromophore and the absorption spectrum of the acceptor chromophore, the lifetime of the excited state, and the relative geometric structure of the transition dipole moments of the chromophores. ${ }^{6}$

For natural photosynthetic systems, there has been evidence of quantum coherence playing an essential role in explaining the observed efficiencies in energy transfer. ${ }^{3,7}$ Quantum coherence occurs due to strong coupling between chromophores leading to a superposition of excited states.

\footnotetext{
a. Institute for Photonics and Advanced Sensing (IPAS) and School of Physical Sciences, The University of Adelaide, Adelaide SA 5005, Australia.

b. Department of Chemistry, Faculty of Science, University of Selcuk, Konya, Turkey, E-mail address: ankursunlu@gmail.com (Ahmed Nuri Kursunlu), Phone: +90 332 2233902 Konya/Turkey

c. Department of Chemistry, The University of Adelaide, Adelaide SA 5005, Australia.

d. Institute of High Performance Computing, Agency of Science, Technology and Research (A*STAR), 1 Fusionopolis Way, \#16-16 Connexis, Singapore 138632, Singapore.
}

Research performed with photosynthetic bacteria has found strong coupling between the bacteria and photons. ${ }^{7,} 8$ Bacteria placed in an optical cavity results in the formation of new eigenstates that are a coherent superposition of photonic and excitonic transitions. ${ }^{8}$ Quantum beats have been observed via two-dimensional electronic spectroscopy (2DES) in the FennaMatthews-Olson bacteriochlorophyll complex found in green sulphur bacteria. ${ }^{7}$

It has been proposed that artificial LHCs mimic photosynthesis. ${ }^{5}$ Because the development of artificial LHCs has been based on those present in nature, inherent similarities between these two forms of LHCs exist in photon absorption and energy transfer. ${ }^{1,}$ 5, 9-11 Specifically, the BODIPY-bearing pillar[5]arene LHC has been shown to exhibit FRET with $92 \%$ efficiency. ${ }^{5}$ Time-dependent density functional theory (TD-DFT) has shown electronic coherence in four chemically unique BODIPY compounds on the order of femtoseconds. ${ }^{12}$

To date, artificial LHCs have not been shown experimentally to exhibit quantum coherence. The development of compounds that exhibit photosynthetic properties analogous to plants and bacteria would allow for the synthesis of solar cells with higher efficiencies in energy transfer, in addition to materials able to store photon energy. The aim of the research presented is to show that the artificial LHC BODIPY pillar[5]arene exhibits quantum coherence via a series of experiments designed to compare light absorption by the LHC to the chromophore.

\section{Methods}

BODIPY - bearing pillar[5]arene synthesis 
All ${ }^{1} \mathrm{H}-\mathrm{NMR}$ and ${ }^{13} \mathrm{C}-\mathrm{NMR}$ spectra of the compounds were carried out on a Varian 400 instrument (standard Trimethylsilane) at $298 \mathrm{~K}$ in $\mathrm{CDCl}_{3}$. The FT-IR spectra of the compounds were collected by a Bruker Fourier Transform Infrared (attenuated total reflection). The elemental analysis and the melting points were measured with Leco CHNS 932 and the Gallenkamp instruments, respectively. Target compounds were synthesised under an argon atmosphere and were prepared using a known literature procedure. ${ }^{5}$ The synthetic

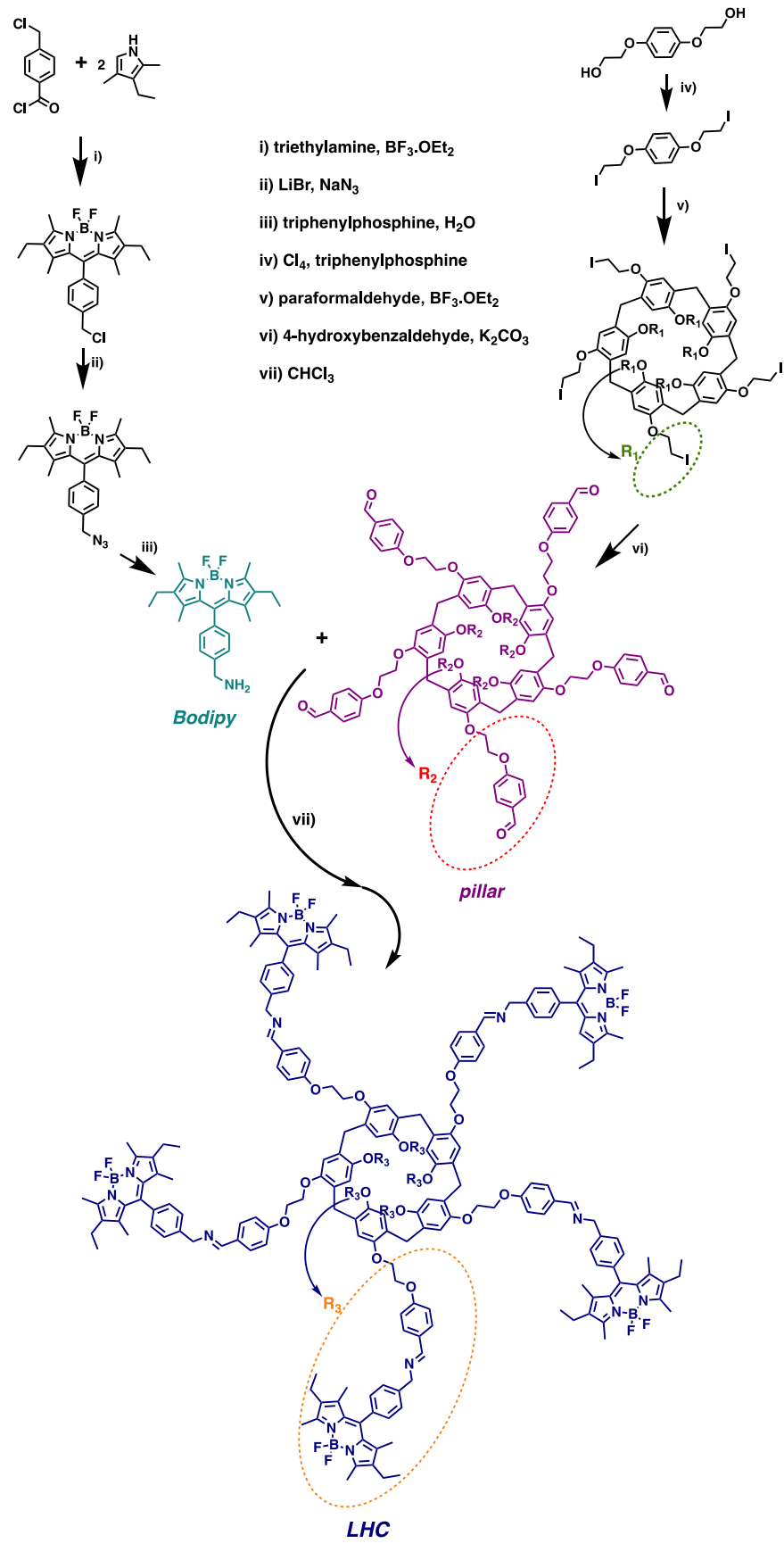

Figure 1 The synthetic route of chromophore, pillar and LHC.

details and data are provided in the electronic supplementary information (ESI). In the synthesis of the chromophore, dichloromethane dried with $\mathrm{CaH}_{2}$ was performed. The completing processes of the reactions were tracked with Merck TLC plates (silica gel 60 on aluminum plate). Triethylamine, lithium bromide, carbon tetraiodide, boron trifluoride ethyl etherate, paraformaldehyde, triphenylphosphine, sodium azide, 4-hydroxybenzaldehyde, and other solvents-reagents were purchased from Sigma-Aldrich, Acros, $\mathrm{TCl}$ Chemicals, Merck, and used without further purification.

\section{Absorption spectroscopy}

After performing a control experiment to show that the absorption spectra of two separate solutions can be summed to give the absorption spectra of the summed concentration (see ESI), solutions of the LHC, chromophore and pillar, each in dichloromethane, were prepared at concentrations of $3 \mu \mathrm{M}, 30$ $\mu \mathrm{M}$ and $3 \mu \mathrm{M}$ respectively. The chosen concentrations ensure the molar ratio of chromophore to pillar (10:1) is satisfied. Each solution was analysed in the Cary 5000 UV-Vis-NIR spectrophotometer.

\section{Transient absorption spectroscopy}

Transient absorption data were collected on a commercial spectrometer (Helios, Ultrafast Systems). The pump and probe laser pulses were sourced from the $800-\mathrm{nm}$ output of a Tisapphire regenerative amplifier (Spitfire Pro XP 100F, Spectra Physics) with $\sim 150$ fs pulse duration and a repetition rate of 1 $\mathrm{kHz}$. The $400-\mathrm{nm}$ pump was produced by frequency doubling in a BBO crystal, with pulse energy of $0.25 \mu \mathrm{J}$ and a spot size of $\sim$ $0.6 \mathrm{~mm}$. No significant power-dependence of the transient absorption dynamics was observed at this excitation density. The white-light probe was generated by focussing a small portion of the amplifier output on to a 3-mm thick sapphire, with a spot size of $\sim 0.15 \mathrm{~mm}$ and a pulse energy $\sim 1000$ times lower than the pump. The polarisation of the pump was rotated to the magic angle $\left(54.7^{\circ}\right)$ relative to the probe to negate effects of anisotropy. Samples were measured in a 2-mm path-length quartz cuvette (21-Q-2, Starna Cells), and diluted in acetone until the maximum absorbance was below 0.6. No photodegradation of the samples was observed over the duration of the experiments.

\section{Broadband pump-probe spectroscopy}

Broadband pump-probe (BBPP) experiments were conducted on a home-built 2-dimensional electronic spectrometer described in detail in a previous publication. ${ }^{13}$ Briefly, the 800 $\mathrm{nm}$ output of the Ti-sapphire regenerative amplifier was used to pump a home-built non-collinear optical parametric amplifier (NOPA) with its central wavelength tuned to $\sim 540 \mathrm{~nm}$. The NOPA output was then compressed using a single-grating and single-prism compressor to give laser pulses close to the transform limit with bandwidth of $27 \mathrm{~nm}$ and a duration of 24 fs (ESI). A diffractive optic element split the beam into the pump and probe. The pump and probe therefore had identical 
characteristics, except that the probe was then attenuated by 4 orders of magnitude using a neutral density filter. The pump arrival time was changed relative to the probe using a pair of rotating optical flats, which allowed for up to $\sim 800 \mathrm{fs}$ of delay with sub-fs precision. The intensity of the probe was detected and spectrally resolved using a spectrograph (Shamrock SR303i, Andor) and CCD camera (Newton 970, Andor). The pump was modulated with a chopper wheel to provide data as the change in probe intensity, $\Delta \mathrm{I}$. Samples were measured in a $1-\mathrm{mm}$ pathlength cuvette (21-Q-1, Starna Cells) and diluted in acetone until the maximum absorbance was below 0.6 .

\section{Computational methods}

To better understand the lowered absorbance upon attaching the chromophore and pillar to the LHC centre, the UV absorption behaviour of these components was probed using computational chemistry methods. Unless otherwise stated, all calculations were carried out using Q-Chem 5.2. ${ }^{14}$ The geometry of isolated fragment molecules (chromophore, pillar, chromophore + pillar) as shown in Table 2 were first optimised using the $\omega$ B97X-D functional with the cc-pVDZ basis set. ${ }^{15}$ The effect of the solvent environment was included using the conductor-like polarisation continuum model (CPCM) with dielectric constant parameter for dichloromethane, $\varepsilon=8.93 .{ }^{16}$ A total of 30 vertical singlet-singlet excitation energies were determined using time-dependent density functional theory (TD-DFT) with the $\omega$ B97X-D functional and the aug-cc-pVDZ basis set, and the corresponding natural transition orbitals (NTOs) were plotted to provide a chemically intuitive picture of the electronic excitation. ${ }^{17}$

\section{Results \& Discussion}

\section{Absorption spectroscopy}

The results obtained from performing UV-Vis absorption spectroscopy on the chromophore, pillar and LHC are illustrated in Figure 2. The absorbance obtained for the LHC and chromophore are of equivalent appearance to those obtained in a previous publication with the presence of a peak at $532 \mathrm{~nm}$ due to the chromophore. ${ }^{5} \mathrm{~A}$ peak is present at $\sim 280 \mathrm{~nm}$ for the pillar compound. This peak is less prominent in the LHC absorption spectrum which is to be expected because the vibrational modes of the pillar-structure in the LHC are different to those of the pillar alone due to the attachment of the chromophores. Figure 2 (b) compares the summed absorption spectra of the pillar and chromophore spectra obtained in (a) to the LHC spectra. Interestingly, a $40 \%$ reduction in absorbance is evident for the LHC compared with the chromophore. The observed reduction in absorbance is more prominent than what was observed for the control experiment discussed in the ESI warranting further investigation into the cause of this reduction.
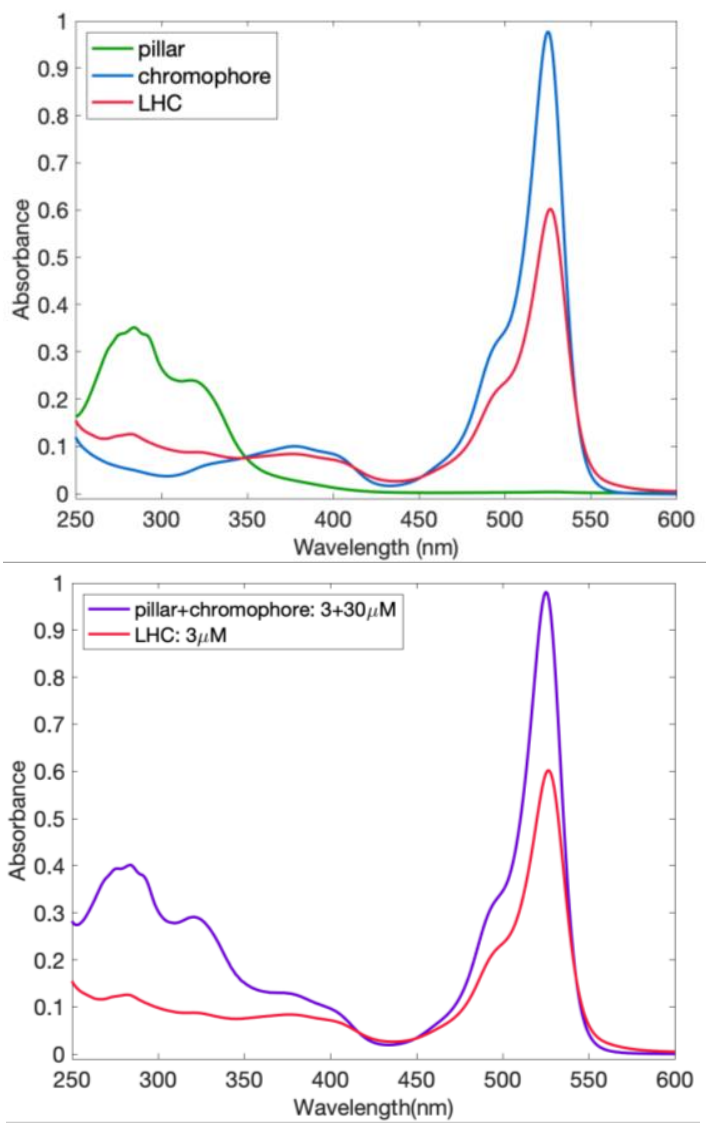

Figure 2 Absorption spectra of pillar and chromophore in (a) are summed to produce the absorption spectra in (b).

\section{Transient absorption spectroscopy}

Figure 3 shows the transient absorption kinetics of both the chromophore and LHC after 400nm excitation. The $525 \mathrm{~nm}$ probe wavelength corresponds to the ground-state bleach band of the molecules, which monitors the depletion of the groundstate population due to the presence of excited or other species. The decay curves are fit with a multi-exponential model, with fit parameters given in Table 1 . The kinetics of the two molecules are quite similar, dominated by a simple exponential decay. Interestingly, the peak magnitude of the bleach signal does not occur immediately after the initial excitation, but evolves in over $\sim 30 \mathrm{ps}$, and is more evident in the kinetics of the chromophore. This is not attributed to spectral evolution, such as red shifting of the absorption peak, as the spectral shape remaining effectively constant over the lifetime of the experiment (ESI). This may be attributed to a solvation effect, where the solvent molecules rearrange due to the changed transition dipole moment of the excited species. ${ }^{18}$ The more crowded nature of the LHC configuration restricts access by solvent molecules to the individual chromophore units, which can explain the reduced effect in the LHC compared to the free chromophores in solution. Recovery from the ground-state bleaching is marginally faster in the LHC, and is consistent with the time-resolved fluorescence data shown in the ESI. 


\begin{tabular}{cccccc} 
Sample & $\lambda(\mathrm{nm})$ & $A_{1}$ & $\tau_{1}(\mathrm{ps})$ & $A_{2}$ & $\tau_{2}(\mathrm{ps})$ \\
\hline chromophore & 525 & $0.11 \pm 0.01$ & $15 \pm 3$ & $-0.89 \pm 0.01$ & $4500 \pm 90$ \\
LHC & 525 & $0.06 \pm 0.02$ & $10 \pm 7$ & $-0.94 \pm 0.01$ & $3540 \pm 90$
\end{tabular}

Table 1 Fitting parameters for the transient absorption data. The data were fit to a multiexponential model of the form $\Delta A(t)=\sum_{n} A_{n} \exp \left(-t / \tau_{n}\right)$, convoluted with a Gaussian instrument response function of $150 \mathrm{fs}$. Amplitudes are normalised so that $\sum_{n}\left|A_{n}\right|=1$.

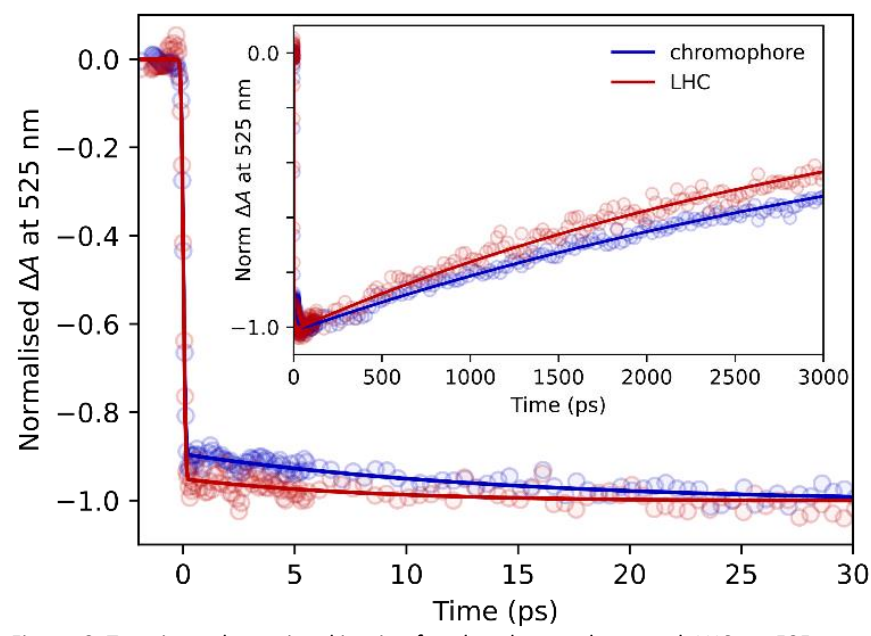

Figure 3 Transient absorption kinetics for the chromophore and LHC at $525 \mathrm{~nm}$ corresponding to the ground-state bleach band. Traces are normalised to their maximum intensity. Solid lines are fits using a multiexponential decay model with parameters shown in Table 1. The inset shows the same data over a larger time window.

The faster decay can be explained by the complex having more non-radiative relaxation pathways, such as vibrational degrees of freedom.

\section{Broadband pump-probe spectroscopy}

Figure 4 and Figure 5 show BBPP data for the chromophore and $\mathrm{LHC}$, respectively. In these experiments, the pump and probe laser pulses are identical, having both short ( $24 \mathrm{fs})$ duration and sufficient bandwidth to excite the molecules to superposition states (ESI). The presence of superposition states is evidenced by oscillations in the signal as a function of time, with a frequency, $\omega$, related to the difference in energy of the states by $E=\hbar \omega$. Frequency analysis of the BBPP data is shown in the ESI.

Note that the oscillatory signals observed in the BBPP data have the same origin as coherences produced in 2DES experiments. Furthermore, BBPP data is often fundamental to the processing of 2DES data to ensure the phasing procedure is performed correctly. ${ }^{19}$ 2DES data collected from the LHC is provided as a video file in the ESI, and exhibit the same oscillations that are observed in the BBPP. Compared to 2DES data, BBPP offers a clear and straightforward method to present coherent oscillations, and thus is used as the focus of this discussion.

The frequency analysis of the BBPP data reveals that both the chromophore and LHC exhibit coherences around $600 \mathrm{~cm}^{-1}$ and $100 \mathrm{~cm}^{-1}$, with no significant differences observed between the two samples. Given that the oscillations are present in the chromophore solution alone, the origin of these coherences can be isolated to the individual chromophore units, rather than interactions between the chromophore units in the LHC, or the chromophore and pillar. The $600 \mathrm{~cm}^{-1}$ frequency is consistent with a vibrational mode of the chromophore core, while the 100 $\mathrm{cm}^{-1}$ signal is likely associated with its substituent groups. ${ }^{20} \mathrm{As}$ the BBPP experiments were carried out at room temperature, any electronic coherences are expected to be very short-lived,
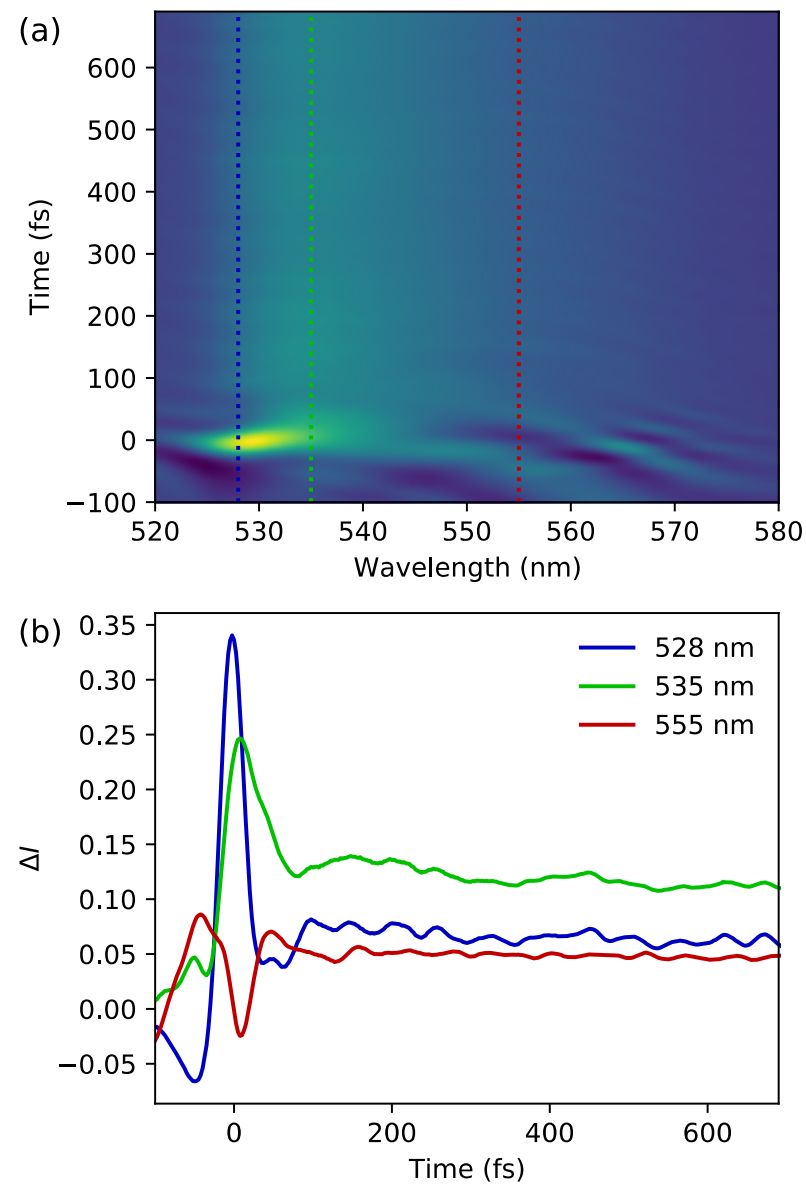

Figure 4 (a) BBPP data for the LHC. The vertical dotted lines correspond to the kinetic slices at selected detection wavelengths shown in (b). 

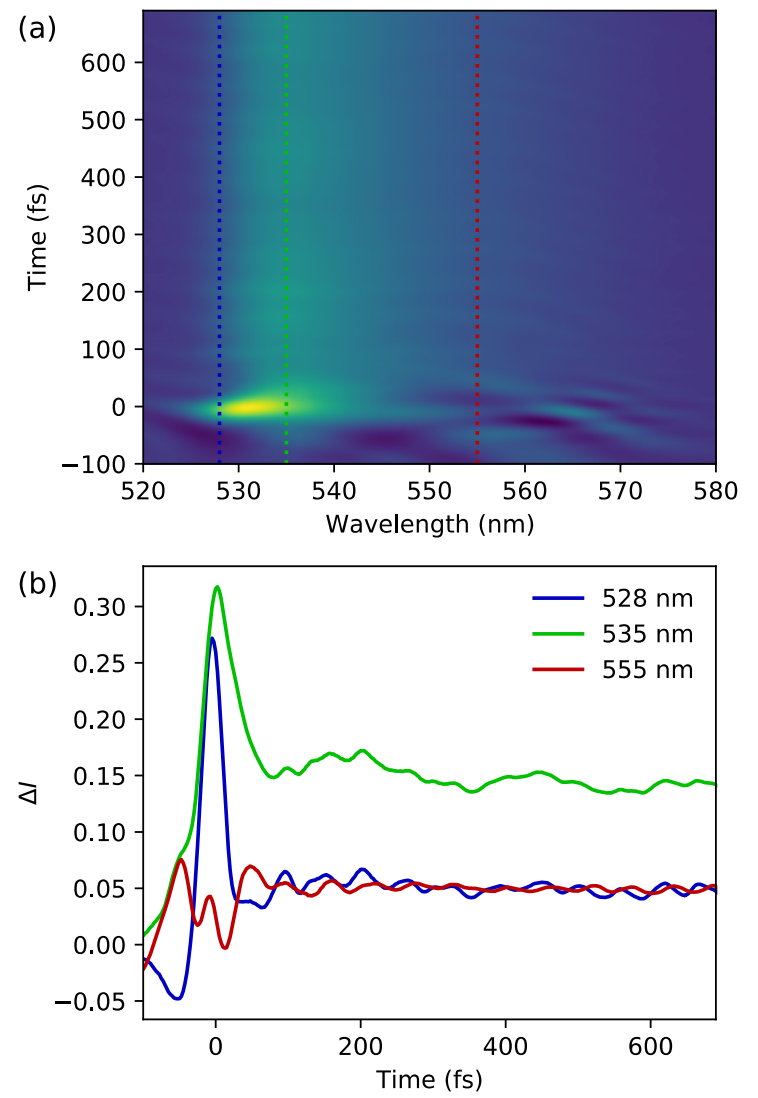

Figure 5 (a) BBPP data for the chromophore. The vertical dotted lines correspond to the kinetic slices at selected detection wavelengths shown in (b).

and not persistent for the ps timescales observed in the data. The observed coherences can therefore be attributed to a vibronic, or exciton-phonon type coupling, where vibrational modes of the molecule are associated with the geometry change between the molecule's ground and excited electronic states. ${ }^{21,} 22$ It should be noted that although very similar BBPP data between the chromophore and LHC were obtained, the presence of electronic couplings between the chromophore units or the pillar remain unclear. The signals associated with these couplings may be outside the detection capabilities of the instrument. The pump and probe laser bandwidth targets the low-energy electronic transitions of the chromophore, covering only the ground-state bleach and stimulated emission bands. The resolution of coherences is limited at high frequencies by the 24-fs laser pulse duration, and at low frequencies by the maximum available pump-probe delay time of $\sim 1 \mathrm{ps}$.

\section{Computational methods}

In order to gain further insight into the inter-chromophore interactions in the LHC, we turn to quantum chemical calculations. The results of the TD-DFT calculation for the isolated chromophore A1, pillar A2, and the chromophore-pillar adduct $\mathrm{B} 1$ are shown in Table 2 . We note that TD-DFT calculated vertical excitation energies for BODIPY dyes are usually overestimated compared to experimental values, however the more accurate SAC-CI and CASPT2 methods are overly demanding for the size of this molecule. ${ }^{23}$ Conventional generalised gradient approximated (GGA) density functionals yield lower mean absolute errors for vertical excitation energies but provide incorrect natural transition orbital (NTOs), thus the $\omega$ B97X-D range-separated hybrid density functional with empirical dispersion corrections was adopted.

The lowest energy excitation in B1 was calculated to be at $418.5 \mathrm{~nm}$, similar to that of the isolated chromophore. There is only one NTO pair for each excitation for B1, with an amplitude above $90 \%$, which indicates little electronic coupling between $A 1$ and $A 2$ when combined. The oscillator strengths for the $416.3 \mathrm{~nm}$ excitation in $\mathrm{A} 1$ and the $418.5 \mathrm{~nm}$ excitation in B1 are 0.8785 and 0.8680 , respectively. Thus, the binding of the pillar to the chromophore moiety should have no effect on the absorbance.

The TD-DFT results for A1, A2 and B1 suggest that the drop in absorbance is unrelated to the coupling of their excited states as the chromophore binds the pillar. We then considered the overall structure of the LHC macromolecule, to see what other effects could influence the non-additivity of UV-Vis absorbance in the chromophore. The overall LHC was constructed and optimised using the GFN2-xTB program, using a semi-empirical tight binding GFN2 Hamiltonian along with the analytical linearised Poisson-Boltzmann solvent model using parameters for dichloromethane as a solvent. ${ }^{24}$ From the optimised structure, four $\pi$-stacked dimeric chromophore moieties can be identified as labelled in Figure 6.

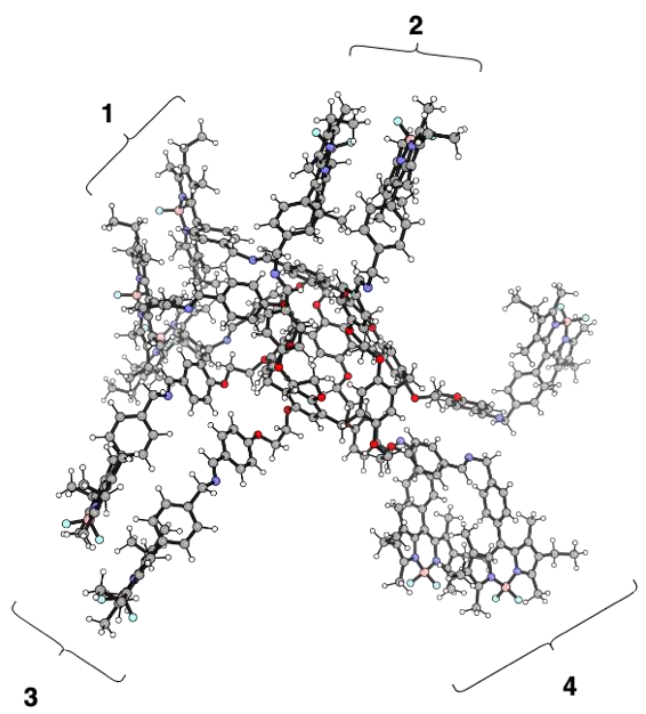

Figure 6 GFN2-xTB optimised structure of LHC, showing four dimeric chromophore moieties.

The structure of dimer 4 was isolated for further TD-DFT analysis to understand the UV-Vis absorption of the LHC theoretically. Methyl groups were used to end-cap the phenoxyl end of the pillar in dimer 4 , and the resultant structure labelled C2 is shown schematically in Figure 7.

The structure of $\mathrm{C} 2$ was then optimised, and TD-DFT calculations carried out in the same manner as used for A1, A2, 
and $\mathrm{B} 1$ to determine the nature of the excitations from the ground state. Results of these calculations are presented in Table 3. A comparison of $\mathrm{B} 1$ and $\mathrm{C} 2$ excitations show that the $418.5 \mathrm{~nm}$ absorption for the chromophore is split into two excitations at $420.8 \mathrm{~nm}$ and $411.1 \mathrm{~nm}$ for the dimer. The oscillator strengths per chromophore for these excitations decrease slightly from 0.8680 for $\mathrm{B} 1$ to 0.0214 and 0.8360 in C2. The $322.3 \mathrm{~nm}$ HOMO-2 $\rightarrow$ LUMO absorption for the chromophore corresponds to two excitations (Exc 3,4 in Table
3) in the dimer, with wavelengths of $324.9 \mathrm{~nm}$ and $323.2 \mathrm{~nm}$. Oscillator strengths decrease significantly from 0.1361 for the chromophore to 0.0059 and 0.0060 for the dimer. The NTOs of these excitations show their charge transfer nature and thus can partially explain the lower absorbance observed experimentally.

\begin{tabular}{|c|c|c|c|c|c|c|}
\hline Molecule & $\lambda / \mathrm{nm}$ & Osc & NTO (hole) & $\begin{array}{l}\text { NTO } \\
\text { (particle) }\end{array}$ & Configuration & Ampl. \\
\hline \multirow[b]{3}{*}{$\begin{array}{l}\text { A1 } \\
\text { chromophore }\end{array}$} & 416.3 & 0.8785 & & & HOMO $\rightarrow$ LUMO & 0.97 \\
\hline & 316.4 & 0.1298 & & & HOMO-1 $\rightarrow$ LUMO & 0.98 \\
\hline & 294.8 & 0.1199 & & & HOMO-3 $\rightarrow$ LUMO & 0.97 \\
\hline \multirow[b]{3}{*}{$\begin{array}{l}\text { A2 } \\
\text { pillar }\end{array}$} & 252.2 & 0.0017 & & & HOMO-2 $\rightarrow$ LUMO & 0.95 \\
\hline & 251.0 & 0.2485 & & & $\begin{array}{l}\text { HOMO-1 } \rightarrow \text { LUMO } \\
\text { HOMO } \rightarrow \text { LUMO } \\
\text { HOMO } \rightarrow \text { LUMO+3 }\end{array}$ & $\begin{array}{l}0.38 \\
0.62 \\
0.62\end{array}$ \\
\hline & 242.0 & 0.5601 & & & $\begin{array}{l}\text { HOMO-1 } \rightarrow \text { LUMO } \\
\text { HOMO } \rightarrow \text { LUMO } \\
\text { HOMO } \rightarrow \text { LUMO+3 }\end{array}$ & $\begin{array}{l}-0.40 \\
0.72 \\
-0.56\end{array}$ \\
\hline \multirow{3}{*}{ chromophore + pillar } & 418.5 & 0.8680 & & & HOMO $\rightarrow$ LUMO & 0.96 \\
\hline & 322.3 & 0.1361 & & & HOMO-2 $\rightarrow$ LUMO & 0.97 \\
\hline & 300.2 & 0.1261 & & & HOMO-3 $\rightarrow$ LUMO & 0.95 \\
\hline
\end{tabular}

Table 2 Vertical excitation wavelengths, oscillator strengths, NTOs (natural transition orbitals), contributing HOMO (highest occupied molecular orbital) and LUMO (lowest unoccupied molecular orbital) configurations and excitation amplitudes for A1 (chromophore), A2 (pillar), and B1 (chromophore + pillar) with significant oscillator strength ( $\mathrm{f}>0.1$ ), calculated using TD-DFT. 

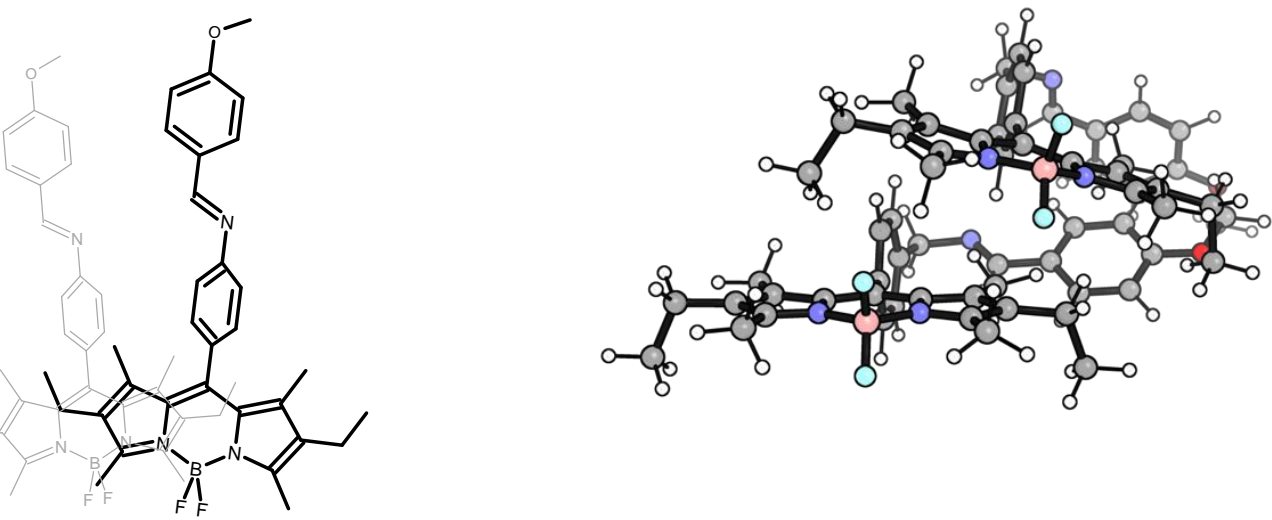

Figure 7 (a) Schematic figure of dimer 4, showing $\pi$-stacking of chromophore groups. Side view of $\pi$-stacked chromophore groups shown in (b).

\begin{tabular}{|c|c|c|c|c|c|c|c|}
\hline Exc & $\lambda / \mathrm{nm}$ & Osc & NTO(h) & NTO(p) & $\begin{array}{l}\text { \% total } \\
\text { ampl. }\end{array}$ & Configurations & Ampl. \\
\hline 1 & 420.8 & $\begin{array}{l}0.0428 \\
(0.0214)\end{array}$ & & & $59.2 \%$ & \multirow{2}{*}{$\begin{array}{l}\text { HOMO- } 1 \rightarrow \text { LUMO } \\
\text { HOMO- } 1 \rightarrow \text { LUMO+1 } \\
\text { HOMO } \rightarrow \text { LUMO } \\
\text { HOMO } \rightarrow \text { LUMO+1 }\end{array}$} & \multirow{2}{*}{$\begin{array}{l}0.6581 \\
0.2283 \\
0.3643 \\
-0.5731\end{array}$} \\
\hline & & & & & $36.9 \%$ & & \\
\hline 2 & 411.1 & $\begin{array}{l}1.6720 \\
(0.8360)\end{array}$ & & & $59.0 \%$ & \multirow{2}{*}{$\begin{array}{l}\text { HOMO- } 1 \rightarrow \text { LUMO } \\
\text { HOMO- } 1 \rightarrow \text { LUMO+1 } \\
\text { HOMO } \rightarrow \text { LUMO } \\
\text { HOMO } \rightarrow \text { LUMO+1 }\end{array}$} & \multirow{2}{*}{$\begin{array}{l}0.4272 \\
-0.4702 \\
0.4404 \\
0.5838\end{array}$} \\
\hline & & & & & $360 \%$ & & \\
\hline 3 & 324.9 & $\begin{array}{l}0.0119 \\
(0.0059)\end{array}$ & & & $99.6 \%$ & $\begin{array}{l}\text { HOMO- } 1 \rightarrow \text { LUMO } \\
\text { HOMO } \rightarrow \text { LUMO }\end{array}$ & $\begin{array}{l}-0.5457 \\
0.7976\end{array}$ \\
\hline 4 & 323.2 & $\begin{array}{l}0.0120 \\
(0.0060)\end{array}$ & & & $99.7 \%$ & $\begin{array}{l}\text { HOMO- } 1 \rightarrow \text { LUMO+1 } \\
\text { HOMO } \rightarrow \text { LUMO+1 }\end{array}$ & $\begin{array}{l}0.8139 \\
0.5311\end{array}$ \\
\hline 5 & 319.0 & $\begin{array}{l}0.0509 \\
(0.0255)\end{array}$ & & & $85.7 \%$ & $\begin{array}{l}\text { HOMO- } 5 \rightarrow \text { LUMO } \\
\text { HOMO- } 4 \rightarrow \text { LUMO }+1\end{array}$ & $\begin{array}{l}0.8768 \\
0.3171\end{array}$ \\
\hline 6 & 317.4 & $\begin{array}{l}0.1646 \\
(0.0823)\end{array}$ & & & $85.6 \%$ & $\begin{array}{l}\text { HOMO- } 5 \rightarrow \text { LUMO } \\
\text { HOMO- } 4 \rightarrow \text { LUMO+1 }\end{array}$ & $\begin{array}{l}-0.3474 \\
0.8824\end{array}$ \\
\hline
\end{tabular}

Table 3 Vertical excitation energies, oscillator strengths (quantities in parenthesis indicates oscillator strength per chromophore), NTOs for first 6 excitations of C2, calculated using TD-DFT. 
(a)

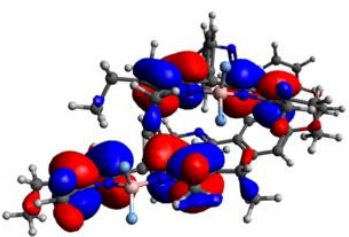

(b)

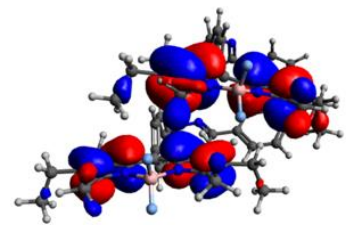

(c)

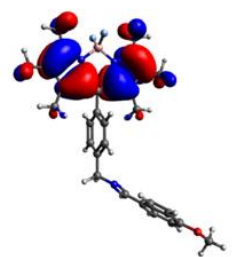

Figure 8 Dimer HOMO (antisymmetric). (b) Dimer HOMO-1 (symmetric). (c) Monomer HOMO

The MO contributions for these NTOs involve the strongly coupled HOMO of both chromophores, which are shown in Figure 8 as a visual guide. Formation of $\pi$-stacked dimers in the overall LHC could result in charge transfer excited states with lowered oscillator strengths that should show up as lowered absorbance.

\section{Conclusion and outlook}

Cavities have been shown to produce and enhance quantum states in photosynthetic bacteria and organic molecules such as Bodipy. ${ }^{8,}$ 25-27 We focused on searching for quantum effects without placing the LHC in a cavity. This work highlights some of the challenges involved with producing artificial LHCs analogous to those found in nature. In the case of the LHC studied in this work, the pillar structure required to place the chromophore units in close proximity resulted in a reduction in absorbance by approximately $40 \%$, which was attributed to hypochromicity due to electronic interactions between the conjugated $\pi$ systems of the chromophores and the pillar. While extending the linkages with non-conjugated units may avoid this issue, the additional geometric flexibility would likely be unfavourable for facilitating stronger coupling between the chromophore units required for coherent energy transport through the system. On the other hand, it is positive to note that other optical properties of the chromophore, such as the absorption spectrum and excited-state lifetime, were effectively unchanged due to the tethering to the pillar structure. This indicates that the properties of an artificial LHC can be engineered primarily through the selection of the chromophore units, with the linkages and backbone playing predominantly only a structural role.

\section{Author Contributions}

SLS: investigation, formal analysis, writing; RK: methodology, investigation; ANK: investigation, visualisation, writing; TWK: investigation, visualisation, writing; РCT: investigation, formal analysis, writing; AMM: investigation, formal analysis, software, visualisation, writing; JQQ: conceptualisation, project administration, methodology, investigation, supervision, writing.

\section{Conflicts of interest}

There are no conflicts to declare.

\section{Acknowledgements}

This work was supported by funding from the Australian Research Council (DP160103797 and LE0989747) and the A*STAR Computational Resource Centre through the use of its high-performance computing facilities. JQQ acknowledges financial support for this work from the Ramsay fellowship.

\section{References}

1. F. Fassioli, R. Dinshaw, P. C. Arpin and G. D. Scholes, Journal of the Royal Society, Interface, 2014, 11, 20130901.

2. Y. Ishida, T. Shimada, D. Masui, H. Tachibana, H. Inoue and S. Takagi, Journal of the American Chemical Society, 2011, 133, 14280

3. E. Wientjes, H. van Amerongen and R. Croce, The Journa of Physical Chemistry. B, 2013, 117, 11200.

4. G. Mcdermott, S. M. Prince, A. A. Freer, A. M. Hawthornthwaite-Lawless, M. Z. Papiz, R. J. Cogdell and N. W. Isaacs, Nature, 1995, 374, 517.

5. A. N. Kursunlu and C. Baslak, Tetrahedron Letters, 2018, 59, 1958-1962.

6. C. J. Law, A. W. Roszak, J. Southall, A. T. Gardiner, N. W. Isaacs and R. J. Cogdell, Molecular Membrane Biology, 2004, 21, 183-191.

7. G. S. Engel, T. R. Calhoun, E. L. Read, T.-K. Ahn, T. Mančal, Y.-C. Cheng, R. E. Blankenship and G. Fleming, R., Nature, 2007, 446, 782.

8. D. Coles, L. C. Flatten, T. Sydney, E. Hounslow, S. K. Saikin A. Aspuru-Guzik, V. Vedral, J. K. H. Tang, R. A. Taylor, J. M. Smith and D. G. Lidzey, Small, 2017, 13, 1701777.

9. T. Nelson, S. Fernandez-Alberti, A. E. Roitberg and S. Tretiak, The Journal of Physical Chemistry Letters, 2017, 8, 3020.

10. C. V. Kumar and J. M. R. Duff, Photochemical \& Photobiological Sciences, 2008, 7, 1522-1530.

11. J. Alster, T. Polívka, J. Arellano, P. Hříbek, F. Vácha, J. Hála and J. Pšenčík, Photosynthesis Research, 2012, 111, 193 204.

12. D. Madrid-Úsuga and J. H. Reina, The Journal of Physical Chemistry A, 2021, 125, 2518-2531. 
13

P. C. Tapping, Y. Song, Y. Kobayashi, G. D. Scholes and T. W Kee, The Journal of Physical Chemistry A, 2020, 124, 10531061.

14. Y. Shao, Z. Gan, E. Epifanovsky, A. T. B. Gilbert, M. Wormit, J. Kussmann, A. W. Lange, A. Behn, J. Deng, X. Feng, D. Ghosh, M. Goldey, P. R. Horn, L. D. Jacobson, I. Kaliman, R. Z. Khaliullin, T. Kuś, A. Landau, J. Liu, E. I. Proynov, Y. M. Rhee, R. M. Richard, M. A. Rohrdanz, R. P. Steele, E. J. Sundstrom, H. L. Woodcock, P. M. Zimmerman, D. Zuev, B. Albrecht, E. Alguire, B. Austin, G. J. O. Beran, Y. A. Bernard, E. Berquist, K. Brandhorst, K. B. Bravaya, S. T. Brown, D. Casanova, C.-M. Chang, Y. Chen, S. H. Chien, K. D. Closser, D. L. Crittenden, M. Diedenhofen, R. A. DiStasio, H. Do, A. D. Dutoi, R. G. Edgar, S. Fatehi, L. Fusti-Molnar, A. Ghysels, A. Golubeva-Zadorozhnaya, J. Gomes, M. W. D. HansonHeine, P. H. P. Harbach, A. W. Hauser, E. G. Hohenstein, Z. C. Holden, T.-C. Jagau, H. Ji, B. Kaduk, K. Khistyaev, J. Kim, J. Kim, R. A. King, P. Klunzinger, D. Kosenkov, T. Kowalczyk, C. M. Krauter, K. U. Lao, A. D. Laurent, K. V. Lawler, S. V. Levchenko, C. Y. Lin, F. Liu, E. Livshits, R. C. Lochan, A. Luenser, P. Manohar, S. F. Manzer, S.-P. Mao, N. Mardirossian, A. V. Marenich, S. A. Maurer, N. J. Mayhall, E. Neuscamman, C. M. Oana, R. Olivares-Amaya, D. P. O’Neill, J. A. Parkhill, T. M. Perrine, R. Peverati, A. Prociuk, D. R. Rehn, E. Rosta, N. J. Russ, S. M. Sharada, S. Sharma, D. W. Small, A. Sodt, T. Stein, D. Stück, Y.-C. Su, A. J. W. Thom, T. Tsuchimochi, V. Vanovschi, L. Vogt, O. Vydrov, T. Wang, M. A. Watson, J. Wenzel, A. White, C. F. Williams, J. Yang, S. Yeganeh, S. R. Yost, Z.-Q. You, I. Y. Zhang, X. Zhang, Y. Zhao, B. R. Brooks, G. K. L. Chan, D. M. Chipman, C. J. Cramer, W. A. Goddard, M. S. Gordon, W. J. Hehre, A. Klamt, H. F. Schaefer, M. W. Schmidt, C. D. Sherrill, D. G. Truhlar, A. Warshel, X. Xu, A. Aspuru-Guzik, R. Baer, A. T. Bell, N. A. Besley, J.-D. Chai, A. Dreuw, B. D. Dunietz, T. R. Furlani, S. R. Gwaltney, C.-P. Hsu, Y. Jung, J. Kong, D. S. Lambrecht, W. Liang, C. Ochsenfeld, V. A. Rassolov, L. V. Slipchenko, J. E. Subotnik, T. Van Voorhis, J. M. Herbert, A. I. Krylov, P. M. W. Gill and M. Head-Gordon, Molecular Physics, 2015, 113, 184-215.

15. J.-D. Chai and M. Head-Gordon, Physical Chemistry Chemical Physics, 2008, 10, 6615-6620.

16. E. Cancès and B. Mennucci, The Journal of Chemical Physics, 2001, 114, 4744-4745.

17. R. L. Martin, The Journal of Chemical Physics, 2003, 118, 4775-4777.

18. J. D. Simon, Accounts of Chemical Research, 1988, 21, 128134.

19. J. M. Anna, Y. Song, R. Dinshaw and G. D. Scholes, Pure and Applied Chemistry, 2013, 85, 1307-1319.

20.

L. Bolzonello, A. Polo, A. Volpato, E. Meneghin, M. Cordaro, M. Trapani, M. Fortino, A. Pedone, M. A. Castriciano and E. Collini, The Journal of Physical Chemistry Letters, 2018, 9, 1079-1085.

21. L. A. Bizimana, J. Brazard, W. P. Carbery, T. Gellen and D. B. Turner, The Journal of Chemical Physics, 2015, 143, 164203.

22. D. B. Turner, K. E. Wilk, P. M. G. Curmi and G. D. Scholes, The Journal of Physical Chemistry Letters, 2011, 2, 1904 1911.

23. M. R. Momeni and A. Brown, Journal of Chemical Theory and Computation, 2015, 11, 2619-2632.
24.

C. Bannwarth, S. Ehlert and S. Grimme, Journal of Chemical Theory and Computation, 2019, 15, 1652-1671.

25. K. Georgiou, P. Michetti, L. Gai, M. Cavazzini, Z. Shen and D. G. Lidzey, ACS Photonics, 2018, 5, 258-266.

26. R. T. Grant, P. Michetti, A. J. Musser, P. Gregoire, T. Virgili, E. Vella, M. Cavazzini, K. Georgiou, F. Galeotti, C. Clark, J. Clark, C. Silva and D. G. Lidzey, Advanced Optical Materials, 2016, 4, 1614-1614.

27. J. Q. Quach, K. E. McGhee, L. Ganzer, D. M. Rouse, B. W. Lovett, E. M. Gauger, J. Keeling, G. Cerullo, D. G. Lidzey and T. Virgili, arXiv, 2020, arXiv:2012.06026. 